\title{
Fluticasone furoate nasal spray: Profile of an enhanced-affinity corticosteroid in treatment of seasonal allergic rhinitis
}

This article was published in the following Dove Press journal:

Journal of Asthma and Allergy

6 August 2010

Number of times this article has been viewed

\author{
Robert Anolik \\ Allergy and Asthma Specialists, \\ PC, Blue Bell, Pennsylvania, USA
}

Correspondence: Robert Anolik Allergy and Asthma Specialists, PC, 470 Sentry Parkway East, Suite 200, Blue Bell, PA 19422, USA

$\mathrm{Tel}+\mathrm{I} 6108255800$

Fax + I 6103970980

Email ranolik@allergypc.com

\begin{abstract}
Of the classes of pharmacotherapy for seasonal allergic rhinitis, intranasal corticosteroids are the preferred treatment and are recommended in practice guidelines as first-line pharmacotherapy for rhinitis with prominent nasal congestion. The enhanced-affinity intranasal corticosteroid fluticasone furoate nasal spray (GW685698X), is one of the newest additions to the armamentarium for allergic rhinitis. This review summarizes the preclinical and clinical data on fluticasone furoate nasal spray and discusses its place in pharmacotherapy for seasonal allergic rhinitis. Important attributes of fluticasone furoate in seasonal allergic rhinitis include low systemic bioavailability $(<0.5 \%)$, onset of symptom relief as early as eight hours after initiation of treatment, 24-hour symptom relief with once-daily dosing, comprehensive coverage of both nasal and ocular symptoms, safety and tolerability with daily use, and availability in a side-actuated device that makes medication delivery simple and consistent. With these attributes, fluticasone furoate nasal spray has the potential to enhance patient satisfaction and compliance and reduce the need for polypharmacy in the management of seasonal allergic rhinitis.
\end{abstract}

Keywords: seasonal allergic rhinitis, allergy, corticosteroid, fluticasone furoate

\section{Introduction}

Allergic rhinitis, which affects approximately one in five Americans, ${ }^{1}$ is an inflammatory disease caused by an IgE-mediated immune response to inhaled allergens at the nasal mucosa. Onset of allergic rhinitis is most common in childhood with a mean age of onset of 8-11 years, but allergic rhinitis occurs in people of all ages. ${ }^{2}$ The disease affects boys more often than girls in childhood, but affects the sexes nearly equally in adulthood. ${ }^{2}$ Allergic rhinitis is characterized by nasal symptoms of congestion, rhinorrhea, sneezing, and ocular symptoms of redness, tearing, and itching, ${ }^{3}$ and it can be complicated by comorbidities including asthma, sinusitis, and otitis media. ${ }^{4}$ Symptoms disturb sleep, cause fatigue, and impair concentration. These effects may underlie the negative effects of allergic rhinitis on productivity and quality of life. ${ }^{1,5-8}$ Each year in the US, an estimated 3.5 million work days and two million school days are lost because of allergic rhinitis, ${ }^{1}$ and estimated annual direct and indirect costs of up to $\$ 4.9$ billion and $\$ 9.7$ billion, respectively, are incurred. ${ }^{9}$

Pharmacotherapy for allergic rhinitis includes intranasal corticosteroids and antihistamine-decongestant combinations, as well as the less commonly used decongestants alone, intranasal anticholinergics, oral, intranasal, and intraocular antihistamines, and mast cell stabilizers. Of these classes of therapy, intranasal corticosteroids are the preferred treatment for seasonal allergic rhinitis and are recommended in both the American Academy of Allergy, Asthma and Immunology 
guidelines and the World Health Organization Allergic Rhinitis and Impact on Asthma guidelines as the first line of pharmacotherapy for rhinitis with prominent nasal congestion, which is the most bothersome symptom of allergic rhinitis. ${ }^{3,10}$ Only intranasal corticosteroids have proven anti-inflammatory activity against the pathophysiologic aspects of both early- and late-phase allergic reactions and are effective for the range of nasal symptoms. ${ }^{10-12}$ In that intranasal corticosteroids inhibit the inflammatory process mediating nasal symptoms and consistently alleviate nasal congestion, they differ from oral antihistamines, which are not particularly effective for nasal congestion. In addition, systemic drug exposure and corresponding risk of systemic side effects are minimal with intranasal corticosteroids.

The enhanced-affinity intranasal corticosteroid fluticasone furoate nasal spray (GW685698X) is one of the newest additions to the armamentarium for allergic rhinitis. Fluticasone furoate was introduced in 2007 as Veramyst ${ }^{\circledR}$ in the US, where it is indicated for treatment of symptoms of seasonal and perennial allergic rhinitis in adults and children two years and older. In Europe, fluticasone furoate is marketed as Avamys ${ }^{\circledR}$ and is indicated for the treatment of symptoms of allergic rhinitis in adults and children six years and older. Fluticasone furoate is a new molecular entity that differs from other corticosteroid molecules, including fluticasone propionate and mometasone furoate. Important attributes of fluticasone furoate in seasonal allergic rhinitis include very low systemic bioavailability $(<0.5 \%)$, onset of symptom relief as early as eight hours after initiation of treatment, 24-hour symptom relief with once-daily dosing, comprehensive coverage of both nasal and ocular symptoms, safety and tolerability with daily use, and availability in a novel, side-actuated delivery device designed to make medication delivery simple and consistent. This review summarizes the preclinical and clinical data on fluticasone furoate nasal spray and discusses its place in pharmacotherapy for allergic rhinitis.

\section{Fluticasone furoate Pharmacology}

Fluticasone furoate is a synthetic fluorinated corticosteroid (Figure 1) with anti-inflammatory effects arising from its interaction with intracellular glucocorticoid receptors. ${ }^{13}$ An enhanced-affinity corticosteroid, fluticasone furoate has greater relative receptor affinity in vitro for the human glucocorticoid receptor than other corticosteroids including dexamethasone, mometasone furoate, fluticasone propionate, ciclesonide active principle, and budesonide (Figure 2). ${ }^{14}$ The precise

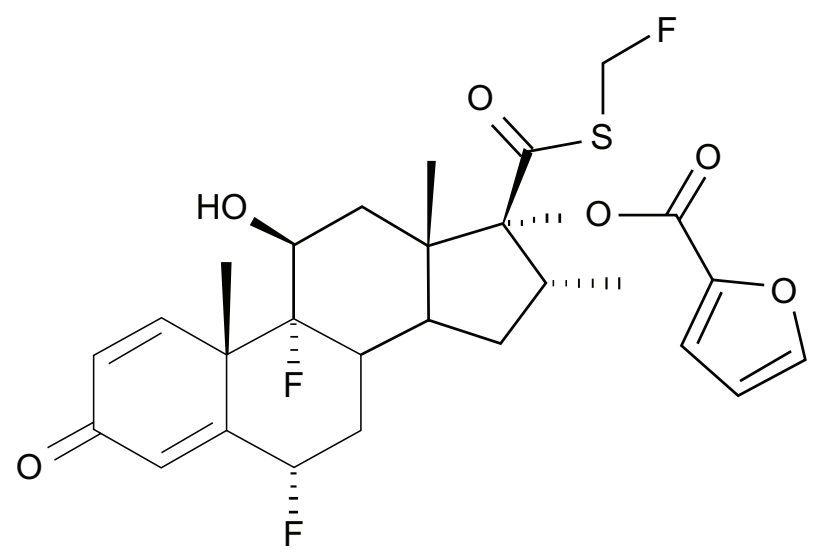

Figure I Chemical structure of fluticasone furoate: $(6 \alpha,|1| \beta,|6 \alpha| ,7 \alpha)$-6,9-difluoroI7-\{[(fluoro-methyl)thio]carbonyl\}-I I-hydroxy-16-methyl-3-oxoandrosta-I,4-dien|7-y| 2-furancarboxylate. ${ }^{15}$

mechanism of action of fluticasone furoate in allergic rhinitis is not definitively known but is thought to arise from one or more of the wide-ranging anti-inflammatory effects it shares with other corticosteroids, which act on multiple inflammatory cell types (eg, mast cells, eosinophils, neutrophils, macrophages, lymphocytes) and affect multiple inflammatory mediators (eg, histamine, eicosanoids, leukotrienes, cytokines)..$^{15}$ In in vitro experimental models, fluticasone furoate demonstrated potent glucocorticoid activity in two pathways, namely the nuclear factor- $\kappa \beta$ pathway and the transactivation glucocorticoid response element pathway, that are downstream of the glucocorticoid receptor and thought to be relevant to anti-inflammatory activity in allergic rhinitis. ${ }^{14}$ In addition, fluticasone furoate potently inhibited the proinflammatory cytokine tumor necrosis factor-alpha and was more effective at preserving epithelial integrity and reducing epithelial permeability in response to protease- and mechanical-induced cell damage than other corticosteroids in vitro. ${ }^{14}$ In studies with isolated human bronchial epithelial cells, fluticasone furoate demonstrated the greatest cellular accumulation and the slowest flux across cellular membranes compared with other corticosteroids for allergic rhinitis but incorporated easily into the cells, properties that are consistent with greater tissue retention and a long duration of action. ${ }^{14}$

\section{Drug delivery device and dosing}

Fluticasone furoate nasal spray is an aqueous suspension of micronized fluticasone furoate for topical administration to the nasal mucosa. It is delivered by a side-actuated, shortnozzle device developed to improve the ease of medication delivery and to minimize variability of the actuated dose. ${ }^{16,17}$ This device was developed based on extensive studies of the preferences of patients and physicians who ranked 


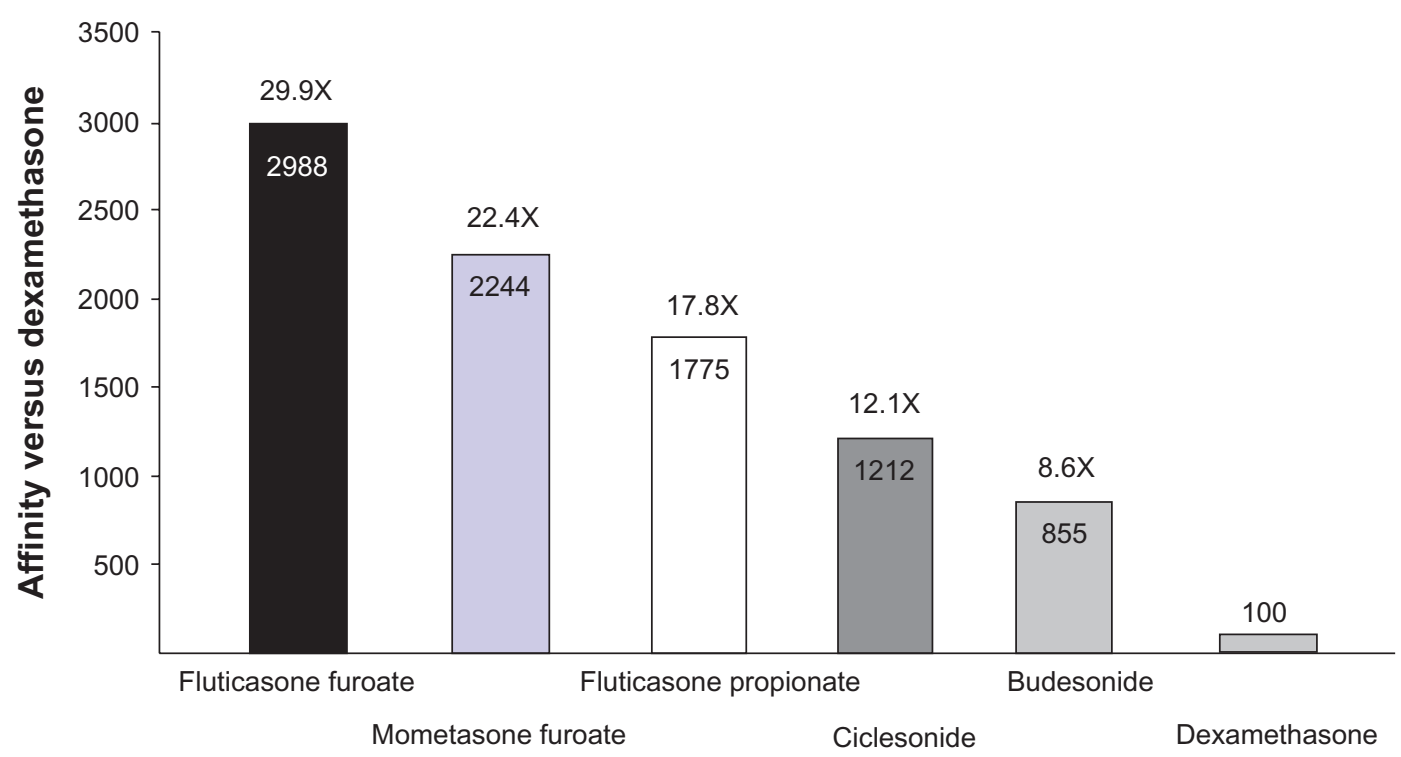

Figure 2 Glucocorticoid receptor affinity versus dexamethasone for corticosteroids administered intranasally. ${ }^{14}$

multiple candidate devices. ${ }^{16}$ The current delivery device was consistently preferred for its design, which renders it easy to hold and operate, and its fine, consistent mist, which facilitates comfortable dispersion of the drug in the nasal cavity. ${ }^{16}$

Each actuation of the device delivers $27.5 \mu \mathrm{g}$ of fluticasone furoate in $50 \mu \mathrm{L}$ of suspension $(0.015 \% \mathrm{w} / \mathrm{w}$ benzalkonium chloride, dextrose anhydrous, edetate disodium, microcrystalline cellulose, carboxymethylcellulose sodium, polysorbate 80 , and purified water). ${ }^{13}$ The device delivers a low spray volume to minimize the amount of drug that runs down the back of the throat or leaks from the nose. Fluticasone furoate had the lowest application volume per spray in a study of several aqueous corticosteroids including budesonide, triamcinolone acetonide, fluticasone propionate, and mometasone furoate. ${ }^{18}$

Fluticasone furoate is administered once daily. According to the US prescribing information, in those 12 years of age and older, the recommended starting dosage is $110 \mu \mathrm{g}$ once daily administered as two sprays $(27.5 \mu \mathrm{g} /$ spray $)$ in each nostril. ${ }^{15}$ In children aged $2-11$ years, the recommended starting dosage in the US is $55 \mu \mathrm{g}$ once daily administered as one spray ( $27.5 \mu \mathrm{g} / \mathrm{spray})$ in each nostril, with the option to increase to $110 \mu \mathrm{g}$ (two sprays in each nostril) once daily in the event of inadequate response to $55 \mu \mathrm{g}$ once daily.

\section{Pharmacokinetics}

Fluticasone furoate nasal spray has low systemic exposure. Because the minimal amount of fluticasone furoate swallowed after intranasal administration is subject to extensive firstpass metabolism, systemic bioavailability is negligible. ${ }^{13}$
Fluticasone furoate administered intranasally to 16 healthy volunteers at supratherapeutic dosages of $880 \mu \mathrm{g}$ at eight-hour intervals for 10 doses, or $2640 \mu \mathrm{g}$ /day, had a geometric mean absolute bioavailability of $0.50 \%$ ( $90 \%$ confidence interval [CI] $0.34 \%-0.74 \%) .{ }^{19}$ Except in isolated cases, fluticasone furoate is not quantifiable in plasma following intranasal dosing of $110 \mu \mathrm{g}$ once daily. ${ }^{13,15}$ Even after administration of a $2 \mathrm{mg}$ oral dose in a study in healthy volunteers, bioavailability was low (1.6\%) because of extensive first-pass metabolism..$^{20}$

The first-pass metabolism of fluticasone furoate is mediated by cytochrome (CYP) 3A4. ${ }^{15}$ Potent inhibitors of CYP 3A4 have the potential to increase systemic exposure to fluticasone furoate and thereby to increase the risk of systemic side effects, such as adrenal suppression. In a drug interaction study, six of 20 subjects coadministered fluticasone furoate nasal spray and the CYP 3A4 inhibitor ketoconazole (200 mg once daily) for seven days had low, but measurable, levels of fluticasone furoate compared with one of 20 subjects coadministered fluticasone furoate and placebo. ${ }^{15}$ Serum cortisol concentrations were reduced over 24 hours by approximately $5 \%$ in subjects who received ketoconazole with fluticasone furoate compared with subjects who received placebo with fluticasone furoate.

\section{Efficacy}

Fluticasone furoate nasal spray has been demonstrated to be significantly more effective than placebo at relieving symptoms of both seasonal allergic rhinitis and perennial allergic rhinitis in children, adolescents, and adults in double-blind, controlled clinical studies. ${ }^{21-29}$ This section describes efficacy 
data from randomized, double-blind, placebo-controlled studies in seasonal allergic rhinitis in patients sensitized to a variety of pollen types including mountain cedar, ragweed, and grass. In these studies, patients used a diary card to rate the severity of individual nasal symptoms of congestion, rhinorrhea, itching, and sneezing on a four-point scale ranging from 0 (symptom not present) to 3 (symptom difficult to tolerate and interferes with activities of daily living and/or sleeping). Ocular symptoms of redness, itching/burning, and tearing/ watery eyes were assessed using the same four-point scale that was used for nasal symptoms. Patients rated these nasal and ocular symptoms on both a reflective basis each morning and evening and on an instantaneous basis each morning. The reflective rating provided information on symptom severity over the preceding 12 hours whereas the instantaneous rating provided information on symptom severity at the moment of the assessment. The morning predose instantaneous rating reflected symptoms at the end of the 24-hour dosing interval on every treatment day (except the first treatment day) because study medication was administered in the morning. At the final clinic visit in most studies, patients rated their overall response to therapy on a seven-point category scale (significantly improved, moderately improved, mildly improved, no change, mildly worse, moderately worse, and significantly worse). Most studies also included the Rhinoconjunctivitis Quality of Life Questionnaire (RQLQ), administered at the beginning and the end of the treatment period. The RQLQ is a 28-item, self-administered, disease-specific instrument that assesses quality of life over a one-week interval. ${ }^{30}$

The primary endpoint was mean change from baseline in reflective total nasal symptom score in all studies except the active-comparator study involving fluticasone propionate (GlaxoSmithKline protocol FFR100652, Table 1) and the active-comparator studies involving fexofenadine (GlaxoSmithKline protocols FFU109045 and FFU109047, Table 1). ${ }^{21,28}$ In the study comparing fluticasone furoate with fluticasone propionate, the primary endpoint was the sum of three individual symptom scores for sneezing + rhinorrhea + nasal congestion. ${ }^{21}$ These three symptoms are considered to be the major symptoms of allergic rhinitis in Japan, where the study was conducted. In the studies comparing fluticasone furoate with fexofenadine, the primary endpoint was nighttime symptoms score, described further in the Nighttime Symptoms section later in this paper. ${ }^{28}$

\section{Nasal symptoms}

Table 1 summarizes the effects of fluticasone furoate nasal spray on nasal symptoms of seasonal allergic rhinitis in randomized, double-blind, placebo-controlled studies. ${ }^{21-25,28,29}$ Across studies, fluticasone furoate nasal spray $110 \mu \mathrm{g}$ conferred comprehensive coverage of nasal symptoms, with significantly better results than placebo for congestion, rhinorrhea, itching, and sneezing. Fluticasone furoate nasal spray $110 \mu \mathrm{g}$ also conferred consistent coverage of nasal symptoms over time, with significantly better results than placebo for morning, evening, and daily reflective nasal symptom scores throughout the study periods. Onset of significant benefit versus placebo was observed as early as eight hours after initiation of treatment for reflective total nasal symptom scores. Furthermore, administered once daily, fluticasone furoate nasal spray $110 \mu \mathrm{g}$ had sustained efficacy over the 24-hour dosing period, as demonstrated by significantly greater improvement compared with placebo in morning predose instantaneous total nasal symptom scores across studies.

Results for patient-rated overall response to therapy and on the RQLQ were consistent with the symptom scores in demonstrating significant benefits of fluticasone furoate nasal spray versus placebo (Table 1). ${ }^{22-25,28}$ Across trials employing the RQLQ, the improvement in RQLQ score with fluticasone furoate versus placebo met or exceeded the minimally important difference (the threshold for being clinically relevant) of 0.5 points (Table 1 ). In a pooled analysis of four of these double-blind, placebo-controlled trials (GlaxoSmithKline protocols FFR20001, FFR30003, FFR103184, and FFR104861), the least square mean difference between fluticasone furoate and placebo was -0.690 (95\% CI -0.84 to -0.54). ${ }^{31} \mathrm{RQLQ}$ results were not reported in the manuscript based on GlaxoSmithKline protocol FFR $100010^{24}$ (the study of children aged 2-11 years) or in the manuscript based on GlaxoSmithKline protocol FFR100652 $2^{21}$ (conducted in Japan). For GlaxoSmithKline protocols FFU109045 28 and FFU10904728 (comparator studies with fexofenadine), the Nocturnal RQLQ (NRQLQ) a 16-item, disease-specific instrument that assesses nocturnal quality of life over a oneweek interval, was used instead of the RQLQ as reported below.

\section{Ocular symptoms}

Table 1 summarizes the effects of fluticasone furoate nasal spray on ocular symptoms of seasonal allergic rhinitis in randomized, double-blind, placebo-controlled studies. Across prospective studies conducted during different allergy seasons and in different geographic regions throughout Europe and the US, fluticasone furoate nasal spray was significantly better than placebo in improving ocular symptoms of seasonal allergic rhinitis (Table 1). ${ }^{22-25,28}$ Efficacy for ocular symptoms 


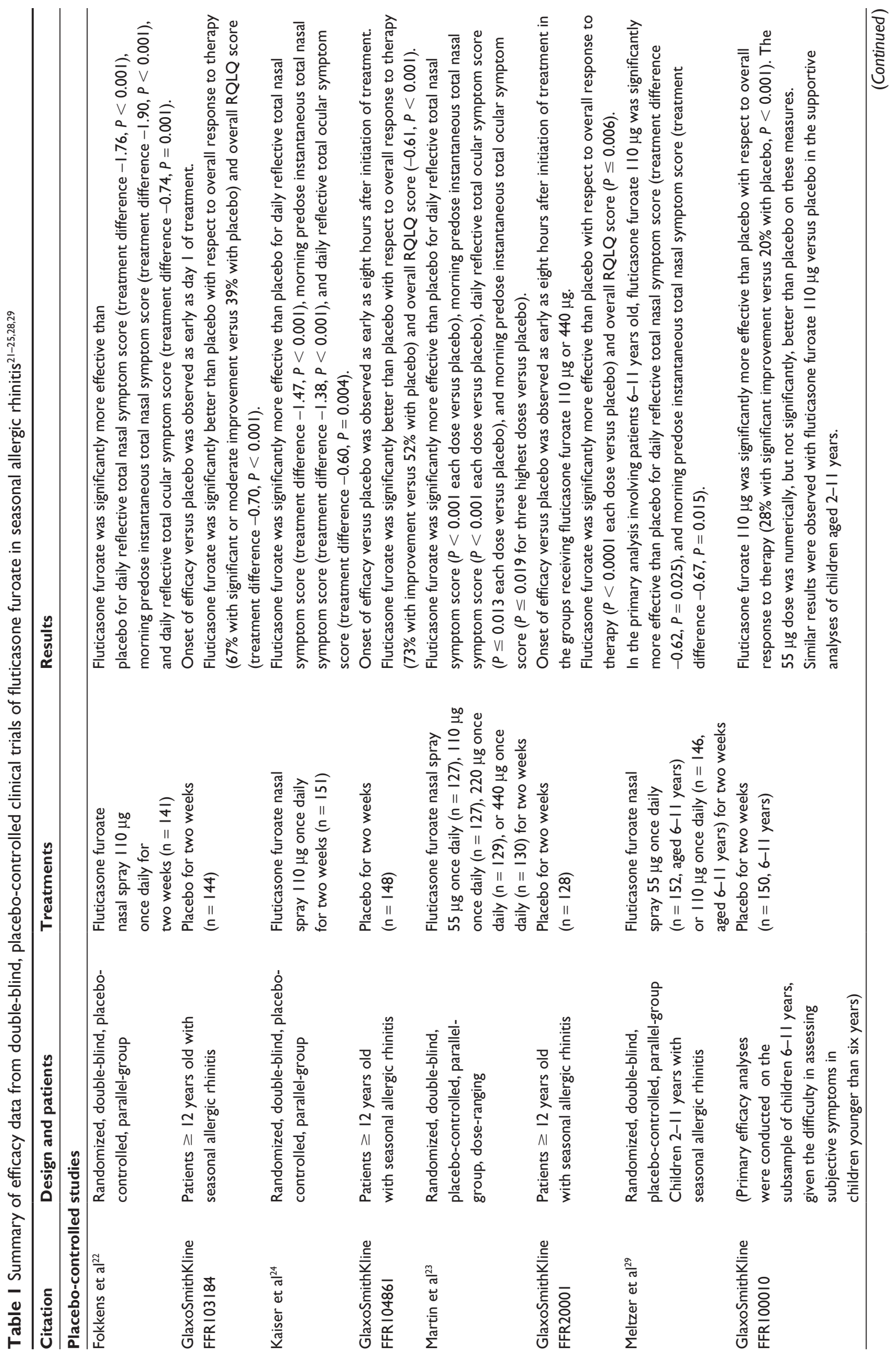




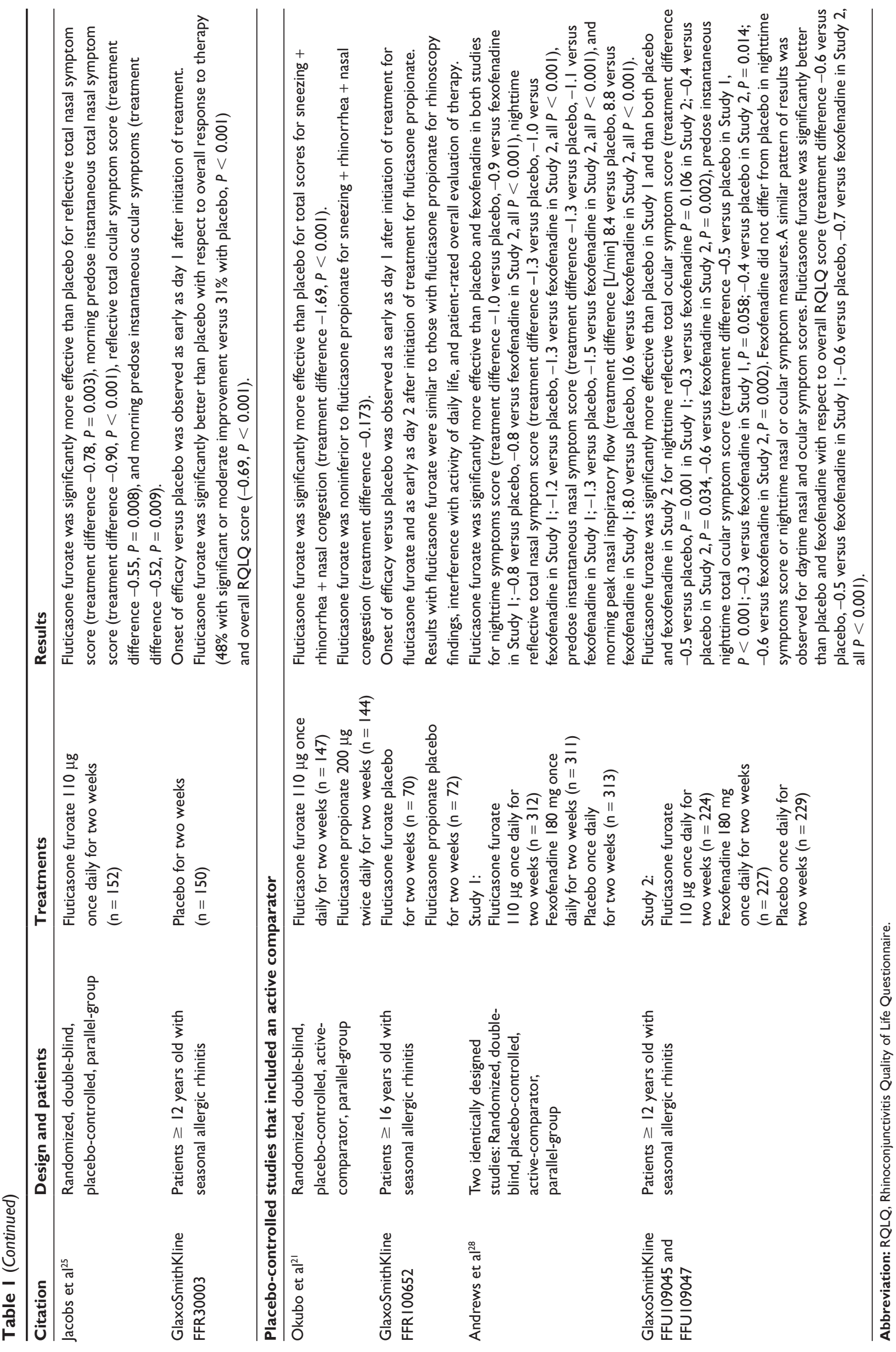


has been observed, but not consistently, with other intranasal corticosteroids. ${ }^{32}$ In a review of 35 randomized, placebo-controlled, clinical trials of seven intranasal corticosteroids in the treatment of seasonal allergic rhinitis, only fluticasone furoate nasal spray demonstrated significant benefit versus placebo on ocular symptoms in prospective studies (6/6 studies). ${ }^{32}$ Beclomethasone dipropionate, budesonide, ciclesonide, fluticasone propionate, mometasone furoate, and triamcinolone acetonide were not consistently effective versus placebo for ocular symptoms across studies. ${ }^{32}$ On the basis of these results, fluticasone furoate nasal spray has been characterized as the only intranasal corticosteroid with consistent efficacy for the ocular symptoms of seasonal allergic rhinitis. ${ }^{31,32}$

The efficacy of fluticasone furoate nasal spray for ocular symptoms is probably not attributed to systemic activity given the extremely low absolute bioavailability $(<0.5 \%)$ of the drug. It has been hypothesized that intranasal corticosteroids improve ocular symptoms of allergic rhinitis by decreasing nasal inflammation and modulating allergen-induced reflex neuronal activity from the nose to the eye. ${ }^{33,34}$ Results of a double-blind, placebo-controlled, crossover study are consistent with this hypothesis. ${ }^{35}$ In 20 patients with seasonal allergic rhinitis, nasal antigen challenge resulted in sneezing and other nasal symptoms, eye symptoms, increases in nasal secretion weights, and increases in percentages of eosinophils in nasal scrapings. Treatment with fluticasone furoate nasal spray reduced eosinophil infiltration and eye symptoms.

The receptor binding properties of fluticasone furoate have been suggested to account for its consistent efficacy for ocular symptoms compared with other intranasal corticosteroids. ${ }^{32}$ Fluticasone furoate has greater affinity for the glucocorticoid receptor than other corticosteroids used intranasally and demonstrates prolonged receptor binding, properties that translate into greater potency than other corticosteroids in models of inflammation. ${ }^{14}$ The greater anti-inflammatory potency of fluticasone furoate as demonstrated in vitro might contribute to more consistent inhibition of the naso-ocular reflex in vivo. ${ }^{32}$

\section{Nighttime symptoms}

Nasal symptoms in allergic rhinitis have been strongly linked to sleeping difficulties and consequent impairment in next-day function and well-being. ${ }^{36-41}$ Pharmacotherapymediated amelioration of nasal symptoms has been linked to improvements in sleep and next-day functioning. ${ }^{36-41}$ The effects of fluticasone furoate nasal spray on nighttime symptoms were assessed in two identically designed, randomized, double-blind, placebo-controlled studies in which patients received fluticasone furoate nasal spray $110 \mu \mathrm{g}$ once daily
( $\mathrm{n}=312$, study $1 ; \mathrm{n}=224$, study 2 ), the oral antihistamine fexofenadine $180 \mathrm{mg}$ daily $(\mathrm{n}=311$, study $1 ; n=227$, study 2$)$, or placebo $(n=313$, study $1 ; n=229$, study 2$)$ for two weeks. ${ }^{28}$ Each morning, patients completed a nighttime symptoms scale comprising three items, ie, nasal congestion upon awakening (rated on a scale from 0 [none] to 3 [severe]), difficulty in going to sleep because of nasal symptoms (rated on a scale from 0 [not at all difficult] to 3 [very difficult]), and nighttime awakenings because of nasal symptoms (rated on a scale from 0 [not at all] to 3 [I feel like I was awake all night]). The sum of scores for the three items is the nighttime symptoms score, a validated, clinically meaningful measure in patients with seasonal allergic rhinitis. ${ }^{42}$ In both studies, fluticasone furoate was significantly more effective $(P<0.001)$ than both placebo and fexofenadine with respect to mean changes from baseline in the nighttime symptoms score. Results for other efficacy endpoints are summarized in Table 1.

The improvements in nighttime symptoms during fluticasone furoate treatment were associated with statistically significant improvements in nocturnal rhinitis-specific quality of life measured with the NRQLQ. ${ }^{43}$ Besides the total NRQLQ score, the scores on each of the NRQLQ domains (sleep problems, sleep time problems, symptoms on waking in the morning, practical problems) were significantly better with fluticasone furoate nasal spray than fexofenadine or placebo.

\section{Summary}

Results of randomized, double-blind, placebo- and comparator-controlled studies demonstrate that fluticasone furoate nasal spray provides comprehensive coverage of both nasal and ocular symptoms in seasonal allergic rhinitis caused by a variety of pollen types. It has also been demonstrated effective for nighttime symptoms of seasonal allergic rhinitis in two randomized, double-blind studies. With once-daily dosing, symptom coverage is maintained throughout the 24-hour dosing interval.

\section{Safety and tolerability}

With intranasal corticosteroids, the occurrence of systemic side effects is limited by the targeted delivery of medication to its nasal mucosal site of action. The low potential of intranasal corticosteroids for causing systemic side effects has been established in both short- and long-term studies. ${ }^{3,11,12}$ In this respect, intranasal corticosteroids differ from oral corticosteroids, which have well documented adverse effects with chronic administration. This section reviews data on the safety and tolerability of fluticasone furoate nasal spray in patients with seasonal allergic rhinitis. Because data in patients with 
perennial allergic rhinitis are also germane to the safety profile of fluticasone furoate nasal spray in seasonal allergic rhinitis, safety results of studies in perennial allergic rhinitis are also discussed when relevant. Studies in perennial allergic rhinitis involved longer treatment periods than studies in seasonal allergic rhinitis and thus constitute a more rigorous test of safety and tolerability than seasonal allergic rhinitis studies.

\section{Adverse events}

The clinical trials program for fluticasone furoate in seasonal and perennial allergic rhinitis provide extensive information on adverse events, defined as any untoward medical occurrence temporally associated with the use of a medicinal product in a patient, regardless of the suspected cause of that occurrence. Adverse event data on fluticasone furoate come from nine placebo-controlled clinical trials of 2-12 weeks' duration in 1563 patients with seasonal or perennial allergic rhinitis. ${ }^{15}$ Six of the nine placebo-controlled trials enrolled 768 adolescents and adults ( 12 years and older) treated with fluticasone furoate $110 \mu \mathrm{g}$ once daily for 2-6 weeks. The remaining three placebo-controlled trials enrolled 795 children (2-11 years) treated with fluticasone furoate $55 \mu \mathrm{g}$ or $110 \mu \mathrm{g}$ once daily for 2-12 weeks. Table 2 summarizes the frequencies of the most common adverse events. Regardless of age group, adverse events were reported with similar frequency with fluticasone

Table 2 Adverse events in placebo-controlled clinical trials of fluticasone furoate in patients with seasonal or perennial allergic rhinitis $^{15}$

\begin{tabular}{|c|c|c|c|}
\hline \multicolumn{4}{|c|}{ Adolescents and adults age $\geq 12$ years in trials lasting 2-6 weeks } \\
\hline & \multicolumn{2}{|c|}{ Fluticasone furoate } & Placebo \\
\hline & \multicolumn{2}{|l|}{$110 \mu \mathrm{g}$} & \\
\hline & \multicolumn{2}{|l|}{$n=768$} & $n=774$ \\
\hline & \multicolumn{2}{|l|}{ n (\%) } & n (\%) \\
\hline Headache & \multicolumn{2}{|l|}{$72(9)$} & $54(7)$ \\
\hline Epistaxis & \multicolumn{2}{|l|}{$45(6)$} & $32(4)$ \\
\hline Pharyngolaryngeal pain & \multicolumn{2}{|l|}{$15(2)$} & $8(1)$ \\
\hline Nasal ulceration & \multicolumn{2}{|l|}{ II (I) } & $3(<1)$ \\
\hline Back pain & \multicolumn{2}{|l|}{$9(1)$} & $7(<1)$ \\
\hline \multicolumn{4}{|c|}{ Pediatric patients aged $2-12$ years in trials lasting $2-12$ weeks } \\
\hline & \multicolumn{2}{|c|}{ Fluticasone furoate } & Placebo \\
\hline & $\begin{array}{l}55 \mu \mathrm{g} \\
\mathrm{n}=369\end{array}$ & $\begin{array}{l}I 10 \mu \mathrm{g} \\
\mathrm{n}=426\end{array}$ & $n=429$ \\
\hline & n (\%) & n (\%) & n (\%) \\
\hline Headache & $28(8)$ & $33(8)$ & $31(7)$ \\
\hline Nasopharyngitis & $20(5)$ & $21(5)$ & $21(5)$ \\
\hline Epistaxis & $17(5)$ & $17(4)$ & $19(4)$ \\
\hline Pyrexia & $17(5)$ & $19(4)$ & $7(2)$ \\
\hline Pharyngolaryngeal pain & $16(4)$ & $12(3)$ & $14(3)$ \\
\hline Cough & $12(3)$ & $16(4)$ & $12(3)$ \\
\hline
\end{tabular}

furoate and placebo. The most common adverse event was headache. Less than $3 \%$ of patients discontinued treatment because of adverse events, and the rate of premature withdrawal from fluticasone furoate was similar to or lower than the rate with placebo.

The adverse event profile of fluticasone furoate nasal spray was assessed in older children, from 6-11 years, in a subanalysis of data from three randomized, double-blind, placebo-controlled, parallel-group studies, including a two-week efficacy and safety study in patients with seasonal allergic rhinitis, a 12-week efficacy and safety study in patients with perennial allergic rhinitis, and a six-week safety study in patients with perennial allergic rhinitis. ${ }^{44}$ Patients were treated with fluticasone furoate $55 \mu \mathrm{g}$ $(\mathrm{n}=297)$, fluticasone furoate $110 \mu \mathrm{g}(\mathrm{n}=321)$, or placebo $(n=330)$. Across studies, the percentages of patients with adverse events considered by the investigator to be drugrelated were $10 \%$ with fluticasone furoate $55 \mu \mathrm{g}, 7 \%$ with fluticasone furoate $110 \mu \mathrm{g}$, and $8 \%$ with placebo. The most common adverse events were headache (8\% with $55 \mu \mathrm{g}$, $9 \%$ with $110 \mu \mathrm{g}, 8 \%$ with placebo), nasopharyngitis (5\% with $55 \mu \mathrm{g}, 6 \%$ with $110 \mu \mathrm{g}, 5 \%$ with placebo), pharyngolaryngeal pain (5\% with $55 \mu \mathrm{g}, 3 \%$ with $110 \mu \mathrm{g}, 4 \%$ with placebo), and epistaxis (4\% with $55 \mu \mathrm{g}, 4 \%$ with $110 \mu \mathrm{g}$, 4\% with placebo).

The adverse event profile of fluticasone furoate nasal spray has also been assessed with long-term daily use. Fluticasone furoate nasal spray was well tolerated in a randomized, doubleblind, placebo-controlled study (GlaxoSmithKline protocol FFR102123) involving 12 months of daily treatment of perennial allergic rhinitis in 605 patients $\geq 12$ years treated with fluticasone furoate $110 \mu \mathrm{g}$ once daily and 201 treated with placebo. ${ }^{45}$ The incidence of most adverse events with fluticasone furoate was similar to that with placebo, with the exception of epistaxis, which was more common with fluticasone furoate $(20 \%)$ than placebo (8\%). Of 123 events of epistaxis in patients treated with fluticasone furoate, 83 were mild, 39 were moderate, and one was severe. ${ }^{15,45}$ All of the 17 events of epistaxis in patients treated with placebo were mild. No evidence of clinically relevant systemic corticosteroid exposure was observed in this study. The incidence of epistaxis in this study was within the range observed with other intranasal corticosteroids (ie, $17 \%-23 \%){ }^{46}$

\section{Hypothalamic-pituitary-adrenal axis function}

Bioavailable exogenous corticosteroids can suppress hypothalamic-pituitary-adrenal axis function. ${ }^{47}$ 
The risk of intranasal corticosteroid-associated hypothalamic-pituitary-adrenal axis suppression is low compared with that associated with the more bioavailable oral corticosteroids. ${ }^{48}$ Potential effects of fluticasone furoate nasal spray on hypothalamic-pituitary-adrenal axis function have been assessed in several studies, none of which demonstrated an effect of the drug. ${ }^{18,49,50}$

The effects of fluticasone furoate nasal spray on hypothalamicpituitary-adrenal axis function as indexed by 24-hour urinary cortisol were assessed in a randomized, double-blind, parallelgroup, placebo-controlled study (GlaxoSmithKline protocol FFR20001). ${ }^{23}$ Patients took fluticasone furoate $55 \mu \mathrm{g}(\mathrm{n}=127)$, $110 \mu \mathrm{g}(\mathrm{n}=127), 220 \mu \mathrm{g}(\mathrm{n}=129), 440 \mu \mathrm{g}(\mathrm{n}=130)$, or placebo $(\mathrm{n}=128)$ once daily for two weeks. At baseline, mean 24-hour urinary cortisol values were comparable among treatment groups and fell within the normal range (ie, $2.1-38 \mu \mathrm{g} / 24$ hours for 12 to 16 -year-olds and $2.0-42.4 \mu \mathrm{g} / 24$ hours for those $>16$ years old) in each treatment group (Table 3). ${ }^{23}$ At the end of the twoweek treatment period, treatment groups did not differ with respect to mean change in 24-hour urinary cortisol, the incidence of patients with values below the lower limit or above the upper limit of normal, or the incidence of shifts to below or above normal values (Table 3 ). ${ }^{23}$
This study, like most of the studies in patients with seasonal allergic rhinitis, involved a two-week treatment period. The effect of fluticasone furoate nasal spray on hypothalamic-pituitary-adrenal axis function have also been assessed in studies with six-week treatment periods in patients with perennial allergic rhinitis. ${ }^{49,50}$ In a randomized, double-blind, parallel-group, placebo- and prednisonecontrolled study in 112 patients 12 years of age and older with perennial allergic rhinitis, patients were administered fluticasone furoate $110 \mu \mathrm{g}$ once daily, prednisone $10 \mathrm{mg}$ once daily, or placebo for six weeks. ${ }^{49}$ Fluticasone furoate was noninferior to placebo with respect to the ratio from baseline in 24-hour serum cortisol weighted mean (treatment ratio $0.98,95 \%$ CI $0.89-1.07$ ) whereas prednisone significantly reduced serum cortisol secretion versus placebo (treatment ratio $0.49,95 \%$ CI $0.43-0.57)$. Fluticasone furoate did not differ from placebo with respect to 24-hour urinary cortisol excretion. In a randomized, double-blind, parallel-group, placebo-controlled study in 112 patients aged 2-11 years with perennial allergic rhinitis, patients were administered fluticasone furoate $110 \mu \mathrm{g}$ once daily or placebo for six weeks. ${ }^{50}$ Fluticasone furoate was noninferior to placebo with respect to the ratio from baseline in 24-hour serum cortisol

Table 3 Summary of data on 24-hour urinary cortisol excretion in the urine cortisol population* in a randomized, double-blind, placebo-controlled, two-week study (GlaxoSmithKline FFR2000I) ${ }^{23}$

\begin{tabular}{|c|c|c|c|c|c|}
\hline & \multicolumn{4}{|c|}{ Fluticasone furoate } & \multirow[t]{2}{*}{ Placebo } \\
\hline & $50 \mu \mathrm{g}$ & $100 \mu \mathrm{g}$ & $200 \mu \mathrm{g}$ & $400 \mu \mathrm{g}$ & \\
\hline \multicolumn{6}{|l|}{ Baseline } \\
\hline $\mathrm{n}$ & 116 & 118 & $|2|$ & 120 & 117 \\
\hline Mean, $\mu g$ /24 hours (SD) & $21.9(18.0)$ & $21.3(21.8)$ & $22.4(15.5)$ & $20.4(12.4)$ & $20.0(14.3)$ \\
\hline \multicolumn{6}{|l|}{ Outside normal range, $\mathrm{n}(\%)$} \\
\hline$>$ Normal & $13(11)$ & $15(13)$ & $12(10)$ & $8(7)$ & $8(7)$ \\
\hline$<$ Normal & $0(0)$ & $5(4)$ & $0(0)$ & $0(0)$ & $\mathrm{I}(\mathrm{I})$ \\
\hline \multicolumn{6}{|l|}{ End of the treatment period } \\
\hline $\mathrm{n}$ & 116 & 118 & 122 & 120 & 117 \\
\hline Mean, $\mu g$ /24 hours (SD) & $23.9(23.1)$ & $20.8(18.3)$ & $21.3(16.4)$ & $20.9(14.9)$ & $20.9(18.7)$ \\
\hline Mean change from baseline (SD) & $2.0(26.3)$ & $-0.5(25.2)$ & $\left(1.2^{\dagger}(19.5)\right.$ & $0.6(17.8)$ & $0.9(20.0)$ \\
\hline \multicolumn{6}{|l|}{ Outside normal range, $\mathrm{n}(\%)$} \\
\hline$>$ Normal & $15(13)$ & $9(8)$ & $16(13)$ & $9(8)$ & II (9) \\
\hline$<$ Normal & I (I) & $2(2)$ & I (I) & $I(I)$ & $I(I)$ \\
\hline \multicolumn{6}{|l|}{ Shifts from baseline, $\mathrm{n}(\%)$} \\
\hline To low & $\mathrm{I}(\mathrm{I})$ & $2(2)$ & $\mathrm{I}(\mathrm{I})$ & $I(I)$ & $\mathrm{I}(\mathrm{I})$ \\
\hline Remained normal or shifted to normal & $104(90)$ & III (94) & $108(89)$ & $112(93)$ & $107(91)$ \\
\hline To high & II (9) & $5(4)$ & $13(11)$ & $7(6)$ & $9(8)$ \\
\hline
\end{tabular}

Note: ${ }^{t} \mathrm{n}=121$.

*The urine cortisol population excluded any patients in the intent-to-treat population who had urine volumes of $<600 \mathrm{~mL}$ for females or $<800 \mathrm{~mL}$ for males and 24 -hour creatinine excretion below the lower limit of the threshold range (defined as mean minus $2.5 \mathrm{SD}$, where the normal range was defined as the mean \pm 2 SD); had a collection time interval outside the range of $24 \pm 4$ hours; used protocol-prohibited systemic or inhaled corticosteroids within eight weeks or protocol-prohibited intranasal or topical corticosteroids within four weeks before any start time of urine collection; and, for the end-of-study urinary cortisol measure, had not taken study medication for more than one day at the start time of urine collection.

Abbreviation: SD, standard deviation. 
weighted mean (treatment ratio 0.97, 95\% CI 0.88-1.07). Fluticasone furoate did not differ from placebo with respect to 24-hour urinary cortisol excretion.

Together, these studies demonstrate that fluticasone furoate nasal spray at doses up to $400 \mu \mathrm{g}$ daily for two weeks or $110 \mu \mathrm{g}$ daily for six weeks did not affect hypothalamicpituitary-adrenal axis function. This result is consistent with the low systemic bioavailability $(<0.5 \%)$ of fluticasone furoate nasal spray.

\section{Growth}

Exogenous corticosteroids can suppress growth via several possible mechanisms, including adrenal suppression with resultant subnormal androgen production, inhibition of growth hormone secretion and insulin-like growth factor-1 bioactivity, and reduction of collagen synthesis. ${ }^{48}$ The risk of intranasal corticosteroid-associated systemic side effects such as growth suppression is low compared with that associated with the more bioavailable oral corticosteroids and older topical preparations such as betamethasone. ${ }^{50,51}$ The effects of fluticasone furoate nasal spray on short-term growth were assessed in 53 prepubertal children (mean age 9.1 years) with seasonal or perennial allergic rhinitis in a randomized, double-blind, placebo-controlled, crossover study. ${ }^{52}$ The two-week fluticasone furoate and placebo treatment periods were separated by a two-week washout period. The primary endpoint was lower leg growth rate assessed by knemometry. Fluticasone furoate did not affect lower leg growth rate versus placebo. Adjusted mean lower leg growth rate was $0.40 \mathrm{~mm} /$ week with fluticasone furoate and $0.42 \mathrm{~mm} /$ week with placebo. The difference between fluticasone furoate and placebo in adjusted mean lower leg growth rate was $-0.016 \mathrm{~mm} /$ week $(95 \% \mathrm{CI}-0.13-0.10)$.

\section{Nasal mucosal effects}

Intranasal administration of corticosteroids is advantageous in that it delivers medication directly to the site of inflammation to maximize relief of nasal symptoms while minimizing the occurrence of systemic side effects. However, it is also potentially detrimental if it results in significant local side effects. The effects of fluticasone furoate nasal spray administered daily for one year on the nasal mucosa were examined in patients with perennial allergic rhinitis in a randomized, open-label study (GlaxoSmithKline protocol FFR104503). ${ }^{53}$ Patients treated with fluticasone furoate $110 \mu \mathrm{g}$ once daily $(\mathrm{n}=37)$ or mometasone furoate nasal spray $200 \mu \mathrm{g}$ once daily $(n=42)$ and a healthy control group $(n=17)$ that did not receive study medication underwent nasal biopsies at baseline and 12 months. The results show that fluticasone furoate nasal spray used daily for one year was not associated with nasal mucosal atrophy. Epithelial thickness did not change appreciably from baseline to 12 months in any group and did not differ between fluticasone furoate and mometasone furoate (least square mean difference $-0.0008 \mathrm{~mm}, 95 \%$ CI $-0.0075-0.0058 ; P=0.802)$.

\section{Summary}

Considered in aggregate, these safety data demonstrate favorable topical and systemic safety of fluticasone furoate nasal spray administered daily for up to one year. The most common adverse events reported in short-term, controlled clinical trials were headache and epistaxis, which were reported with similar frequency for fluticasone furoate and placebo. During long-term treatment, epistaxis, which was most often mild, occurred more frequently with fluticasone furoate than placebo. This adverse event profile is consistent with that of other intranasal corticosteroids. ${ }^{31}$ No adverse effects on hypothalamic-pituitary-adrenal axis function or growth have been demonstrated in studies conducted to date. A placebocontrolled study (clinicaltrials.gov identifier NCT00570492; GlaxoSmithKline protocol FFR101782) is being conducted to assess further the potential effects of fluticasone furoate $110 \mu \mathrm{g}$ once daily for one year on growth in pediatric patients aged 5-8 years with perennial allergic rhinitis.

\section{Medical costs}

Polypharmacy is common in allergic rhinitis. In a survey of 447 patients with allergic rhinitis and their physicians, 56\% reported use of two or more medications to control their allergic rhinitis. ${ }^{6}$ Comprehensive coverage of both nasal and ocular symptoms by fluticasone furoate nasal spray could potentially reduce the need for polypharmacy relative to agents that cover only nasal symptoms. The reduced need for polypharmacy would be expected to translate into a reduction in medical costs. This hypothesis was tested in a retrospective cohort analysis of pharmacy claims data from 793,349 patients with at least one claim for fluticasone furoate, budesonide, mometasone furoate, or triamcinolone acetonide from 1 April to 31 July $2007 .{ }^{54}$ At index, $62.9 \%$ of patients were using mometasone furoate, $21.1 \%$ triamcinolone acetonide, $15.1 \%$ budesonide, and $1.0 \%$ fluticasone furoate. (The low rate of use of fluticasone furoate is attributed to the fact that the study period occurred just after the drug's introduction in the US in 2007.) Patients treated with fluticasone furoate compared with the other intranasal corticosteroids were $21 \%$ less likely to use concomitant prescription allergic rhinitis drugs (other 
than intranasal corticosteroids) and incurred significantly lower costs of concomitant allergic rhinitis drugs. The authors concluded that fluticasone furoate compared with the other intranasal corticosteroids in the study reduced the need for concomitant prescription allergic rhinitis medications and led to lower costs per patient with potentially significant savings for health plans. ${ }^{54}$

\section{Patient perceptions vis-à-vis compliance}

Patients' self-reported willingness to comply with nasal spray therapy is strongly affected by several factors, including efficacy, safety, ease of use, comfort during administration, and sensory attributes such as the smell, taste, and aftertaste of a nasal spray. ${ }^{16,55}$ In a qualitative study involving one-on-one interviews with 24 physicians and 25 patients with allergic rhinitis, features identified as strengths of fluticasone furoate nasal spray included its appearance; its design, which makes it easier to hold and operate; side actuation, which facilitates control during drug administration; the fine, consistent mist; and the delivery nozzle, which feels comfortable in the nose. ${ }^{16}$ The profile of fluticasone furoate nasal spray addresses many of these factors. First, as discussed above, fluticasone furoate nasal spray is effective and generally well tolerated with once daily use. Second, the fluticasone furoate delivery device, was designed to maximize ease of use and comfort during administration. These results are supported by qualitative assessments of the delivery device in patients in clinical trials. ${ }^{16}$ Third, data suggest that the sensory attributes of fluticasone furoate also are conducive to patient compliance. In a crossover comparison of fluticasone furoate nasal spray and fluticasone propionate nasal spray in 127 patients with allergic rhinitis, patients preferred fluticasone furoate nasal spray over fluticasone propionate based on the attributes of odor ( $64 \%$ versus $29 \%$ ), taste ( $47 \%$ versus $21 \%$ ), aftertaste ( $44 \%$ versus $22 \%$ ), drip down the throat ( $43 \%$ versus $27 \%$ ), and nose runoff $(49 \%$ versus $19 \%)^{56}$

Compliance rates with fluticasone furoate nasal spray have not been systematically assessed in clinical practice, nor have compliance rates with fluticasone furoate nasal spray been directly compared with those of other intranasal corticosteroids. In clinical trials in which patients with perennial allergic rhinitis were assigned to administer fluticasone furoate nasal spray or placebo once daily for six weeks, mean compliance rates ranged from $86 \%$ to $95 \%{ }^{26,27}$ In the 12-month, placebo-controlled safety study in patients with perennial allergic rhinitis (GlaxoSmithKline protocol FFR102123), 85\% of fluticasone furoate-treated patients had $\geq 90 \%$ compliance, and an additional $10 \%$ had $\geq 80 \%-90 \%$ compliance. ${ }^{46}$ In these studies, compliance was assessed by patients' indicating in a daily diary whether they had administered two sprays of study medication into each nostril on each treatment day. Compliance was calculated for each patient as the number of days on which double-blind study medication was taken divided by the number of days of exposure to study treatment. Future research comparing compliance with fluticasone furoate with that of other intranasal corticosteroids is warranted in light of the putative compliance-enhancing properties of fluticasone furoate.

\section{Conclusions}

The important attributes of fluticasone furoate in seasonal allergic rhinitis include low systemic bioavailability $(<0.5 \%)$, onset of symptom relief as early as eight hours after initiation of treatment, 24-hour symptom relief with once-daily dosing, comprehensive coverage of both nasal and ocular symptoms, safety and tolerability with daily use, and availability in a device that could help enhance medication adherence. Fluticasone furoate nasal spray meets the present need for an intranasal corticosteroid with consistent efficacy for ocular symptoms in seasonal allergic rhinitis. Given the frequency and bothersome nature of ocular symptoms in allergic rhinitis, ${ }^{31}$ the consistent efficacy of fluticasone furoate nasal spray for ocular symptoms could prove to be important in reducing the need for polypharmacy, as suggested by the cohort analysis described earlier. ${ }^{54}$ Furthermore, with a delivery device that improves the experience of medication delivery, fluticasone furoate could also prove to be unique in enhancing patient satisfaction and compliance.

\section{Acknowledgments}

The author acknowledges Jane Saiers, PhD (The WriteMedicine, Inc.) for assistance with writing the manuscript, creating tables and figures, and doing literature searches for the manuscript.

\section{Disclosure}

This work was funded by GlaxoSmithKline.

\section{References}

1. Nathan RA. The burden of allergic rhinitis. Allergy Asthma Proc. 2007; 28:3-9.

2. Sheikh J, Najib U. Allergic rhinitis. emedicine. 2009 Jun 16. Available at: http://emedicine.medscape.com/article/134825-overview. Accessed Dec 8, 2009.

3. Dykewicz MS, Fineman S. Executive summary of Joint Task Force Practice Parameters on Diagnosis and Management of Rhinitis. Ann Allergy Asthma Immunol. 1998;81:463-468.

4. Skoner DP. Complications of allergic rhinitis. J Allergy Clin Immunol. 2000;105:S605-S609. 
5. Meltzer EO, Nathan R, Derebery J, et al. Sleep, quality of life, and productivity impact of nasal symptoms in the United States: Findings from the Burden of Rhinitis in America survey. Allergy Asthma Proc. 2009:30:244-254.

6. Schatz M. A survey of the burden of allergic rhinitis in the USA. Allergy. 2007;62 Suppl 85:9-16.

7. Juniper EF. Measuring health-related quality of life in rhinitis. JAllergy Clin Immunol. 1997;99:S742-S749.

8. Ferguson BJ. Influences of allergic rhinitis on sleep. Otolaryngol Head Neck Surg. 2004;130:617-629.

9. Reed SD, Lee TA, McCrory DC. The economic burden of allergic rhinitis: A critical evaluation of the literature. Pharmacoeconomics. 2004;22:345-361.

10. Task Force on Allergic Disorders - American Academy of Allergy, Asthma, and Immunology. The Allergy Report. Available at: www. theallergyreport.org. Accessed Mar 4, 2005.

11. Weiner JM, Abramson MJ, Puy RM. Intranasal corticosteroids versus oral $\mathrm{H}_{1}$ receptor antagonists in allergic rhinitis: Systematic review of randomised controlled trials. BMJ. 1998;317:1624-1629.

12. Stempel DA, Thomas M. Treatment of allergic rhinitis: An evidence-based evaluation of nasal corticosteroids versus nonsedating antihistamines. Am J Manag Care. 1998;4:89-96.

13. Giavina-Bianchi P, Agondi R, Stelmach R, Cukier A, Kalil J. Fluticasone furoate nasal spray in the treatment of allergic rhinitis. Ther Clin Risk Manag. 2008;4:465-472.

14. Salter M, Biggadike K, Matthews JL, et al. Pharmacological properties of the enhanced-affinity glucocorticoid fluticasone furoate in vitro and in an in vivo model of respiratory inflammatory disease. Am J Physiol Lung Cell Mol Physiol. 2007;293:L660-L667.

15. Veramyst (fluticasone furoate) Nasal Spray. Full prescribing information. 2009, GlaxoSmithKline. Available at: http://us.gsk.com/products/ assets/us_veramyst.pdf. Accessed Dec 1, 2009.

16. Berger WE, Godfrey JW, Slater AL. Intranasal corticosteroids: The development of a drug delivery device for fluticasone furoate as a potential step toward improved compliance. Expert Opin Drug Deliv. 2007;4:689-701.

17. McCormack PL, Scott LJ. Fluticasone furoate: Intranasal use in allergic rhinitis. Drugs. 2007;67:1905-1915.

18. Baumann D, Bachert C, Hogger P. Dissolution in nasal fluid, retention and anti-inflammatory activity of fluticasone furoate in human nasal tissue ex vivo. Clin Exp Allergy. 2009;39:1540-1550.

19. Allen A, Down G, Newland A, et al. Absolute bioavailability of intranasal fluticasone furoate in healthy subjects. Clin Ther. 2007;29: $1415-1420$

20. Hughes SC, Shardlow PC, Hollis FJ, et al. Metabolism and disposition of fluticasone furoate, an enhanced-affinity glucocorticoid, in humans. Drug Metab Dispos. 2008;36:2337-2344.

21. Okubo K, Nakashima M, Miyake N, Komatsubara M, Okuda M. Comparison of fluticasone furoate and fluticasone propionate for the treatment of Japanese cedar pollinosis. Allergy Asthma Proc. 2009;30:84-94.

22. Fokkens WJ, Jogi R, Reinartz S, et al. Once daily fluticasone furoate nasal spray is effective in seasonal allergic rhinitis caused by grass pollen. Allergy. 2007;62:1078-1084.

23. Martin BG, Ratner PH, Hampel FC, et al. Optimal dose selection of fluticasone furoate nasal spray for the treatment of seasonal allergic rhinitis in adults and adolescents. Allergy Asthma Proc. 2007;28: 216-225.

24. Kaiser HB, Naclerio RM, Given J, Toler TN, Ellsworth A, Philpot EE. Fluticasone furoate nasal spray: A single treatment option for the symptoms of seasonal allergic rhinitis. J Allergy Clin Immunol. 2007; 119:1430-1437.

25. Jacobs R, Martin B, Hampel F, Toler TN, Ellsworth A, Philpot EE. Effectiveness of fluticasone furoate $110 \mathrm{mcg}$ once daily in the treatment of nasal and ocular symptoms of seasonal allergic rhinitis in adults and adolescents sensitized to mountain cedar pollen. Curr Med Res Opin. 2009;25:1393-1401.
26. Nathan RA, Berger W, Yang W, et al. Effect of once-daily fluticasone furoate nasal spray on nasal symptoms in adults and adolescents with perennial allergic rhinitis. Ann Allergy Asthma Immunol. 2008;100:497-505.

27. Vasar M, Houle PA, Douglass JA, et al. Fluticasone furoate nasal spray: effective monotherapy for symptoms of perennial allergic rhinitis in adults/adolescents. Allergy Asthma Proc. 2008;29:313-321.

28. Andrews CP, Martin BG, Jacobs RL, et al. Fluticasone furoate nasal spray is more effective than fexofenadine for nighttime symptoms of seasonal allergy. Allergy Asthma Proc. 2009;30:128-138.

29. Meltzer EO, Lee J, Tripathy I, Lim J, Ellsworth A, Philpot E. Efficacy and safety of once-daily fluticasone furoate nasal spray in children with seasonal allergic rhinitis treated for 2 weeks. Pediatr Allergy Immunol. 2009:20:279-286.

30. Juniper EF, Thompson AK, Ferrie PJ, Roberts JN. Validation of the standardised version of the Rhinoconjunctivitis Quality of Life Questionnaire. J Allergy Clin Immunol. 1999;104:364-369.

31. Scadding GK, Keith PK. Fluticasone furoate nasal spray consistently and significantly improves both the nasal and ocular symptoms of seasonal allergic rhinitis: A review of the clinical data. Expert Opin Pharmacother. 2008;9:2707-2715.

32. Keith PK, Scadding GK. Are intranasal corticosteroids all equally consistent in managing ocular symptoms of seasonal allergic rhinitis? Curr Med Res Opin. 2009;8:2021-2041.

33. DeWester J, Philpot EE, Westlund RE, Cook CK, Rickard KA. The efficacy of intranasal fluticasone propionate in the relief of ocular symptoms associated with seasonal allergic rhinitis. Allergy Asthma Proc. 2003;24:331-337.

34. Bernstein DI, Levy AL, Hampel FC, et al. Treatment with intranasal fluticasone propionate significantly improves ocular symptoms in patients with seasonal allergic rhinitis. Clin Exp Allergy. 2004;34:952-957.

35. Baroody FM, Shenag D, DeTineo M, et al. Fluticasone furoate nasal spray reduces the nasal-ocular reflex: A mechanism for the efficacy of topical steroids in controlling allergic eye symptoms. J Allergy Clin Immunol. 2009;123:1342-1348.

36. Bender BG. Cognitive effects of allergic rhinitis and its treatment. Immunol Allergy Clin North Am. 2005;25:301-312.

37. Pratt EL, Craig TJ. Assessing outcomes from the sleep disturbance associated with rhinitis. Curr Opin Allergy Clin Immunol. 2007;7:249-256.

38. Léger D, Annesi-Maesano I, Carat F, et al. Allergic rhinitis and its consequences on quality of sleep. Arch Intern Med. 2006;166;1744-1748.

39. Green RJ, Davis G, Price D. Concerns of patients with allergic rhinitis: The Allergic Rhinitis Care Programme in South Africa. Prim Care Respir J. 2007;16:299-303.

40. Gurevich F, Glass C, Davies M, et al. The effect of intranasal steroid budesonide on the congestion-related sleep disturbance and daytime somnolence in patients with perennial allergic rhinitis. Allergy Asthma Proc. 2005;26:268-274

41. Craig TJ, Hanks CD, Fisher LH. How do topical nasal corticosteroids improve sleep and daytime somnolence in allergic rhinitis? J Allergy Clin Immunol. 2005;116:1264-1266.

42. Santanello NC, DeMuro-Mercon C, Shah SR, et al. Validation of the nighttime symptoms score as a clinically relevant measure of allergic rhinitis. Allergy Asthma Proc. 2006;27:231-239.

43. Juniper EF, Rohrbaugh T, Meltzer EO. A questionnaire to measure quality of life in adults with nocturnal allergic rhinoconjunctivitis. J Allergy Clin Immunol. 2003;111:484-490.

44. Meltzer EO, Tripathy I, Maspero JF, Wu W, Philpot E. Safety and tolerability of fluticasone furoate nasal spray once daily in paediatric patients aged 6-11 years with allergic rhinitis: Subanalysis of three randomized, double-blind, placebo-controlled, multicentre studies. Clin Drug Investig. 2009;29:79-86.

45. Rosenblut A, Bardin PG, Muller B, et al. Long-term safety of fluticasone furoate nasal spray in adults and adolescents with perennial allergic rhinitis. Allergy. 2007;1071-1077.

46. Waddell AN, Patel SK, Toma AG, Maw AR. Intranasal steroid sprays in the treatment of rhinitis: Is one better than another? J Laryngol Otol. 2003;117:843-845. 
47. Schimmer BP, Parker KL. Adrenocorticotropic hormone; adrenocortical steroids and their synthetic analogs; inhibitors of the synthesis and actions of adrenocortical hormones. In: Hardman JG, Limbird LE editors. Goodman and Gilman's The Pharmacological Basis of Therapeutics. 9th ed. New York, NY: McGraw-Hill, 1996.

48. Allen DB. Systemic effects of intranasal steroids: An endocrinologist's perspective. J Allergy Clin Immunol. 2000;106:S179-S190.

49. Patel D, Ratner P, Clements D, Wu W, Faris M, Philpot E. Lack of effect on adult and adolescent hypothalamic-pituitary-adrenal axis function with use of fluticasone furoate nasal spray. Ann Allergy Asthma Immunol. 2008; 100:490-496.

50. Tripathy I, Levy A, Ratner P, Clements D, Wu W, Philpot E. HPA axis safety of fluticasone furoate nasal spray once daily in children with perennial allergic rhinitis. Pediatr Allergy Immunol. 2009;20: 287-294.

51. Scadding GK. Corticosteroids in the treatment of pediatric allergic rhinitis. J Allergy Clin Immunol. 2001;108:S59-S64.

52. Gradman J, Caldwell MF, Wolthers OD. A 2-week, crossover study to investigate the effect of fluticasone furoate nasal spray on shortterm growth in children with allergic rhinitis. Clin Ther. 2007;29: 1738-1747.
53. Clinical Study ID FFU104503. A randomized, open label, active controlled (mometasone furoate aqueous nasal spray [Nasonex ${ }^{\circledR}$ ] $200 \mathrm{mcg}$ QD), parallel group, multi-center, 52-week study to assess the long term safety of GW685698X aqueous nasal spray $100 \mathrm{mcg} *$ QD via nasal biopsy in subjects $>/ 18$ years of age with perennial allergic rhinitis. Available at: www.gsk-clinicalstudyregister.com. Accessed Dec $11,2009$.

54. Garris C, Shah M, D'Souza A, Stanford R. Comparison of corticosteroid nasal sprays in relation to concomitant use and cost of other prescription mediations to treat allergic rhinitis symptoms: Retrospective cohort analysis of pharmacy claims data. Clin Drug Investig. 2009;29: 515-526.

55. Mahadevia PJ, Shah S, Leibman C, Kleinman L, O’Dowd L. Patient preferences for sensory attributes of intranasal corticosteroids and willingness to adhere to prescribed therapy for allergic rhinitis: A conjoint analysis. Ann Allergy Asthma Immunol. 2004;93:345-350.

56. Meltzer EO, Stahlman JE, Leflein J, et al. Preferences of adult patients with allergic rhinitis for the sensory attributes of fluticasone furoate versus fluticasone propionate nasal sprays: A randomized, multicenter, double-blind, single-dose, crossover study. Clin Ther. 2008;30: $271-279$.

\section{Publish your work in this journal}

The Journal of Asthma and Allergy is an international, peer-reviewed open-access journal publishing original research, reports, editorials and commentaries on the following topics: Asthma; Pulmonary physiology; Asthma related clinical health; Clinical immunology and the immunological basis of disease; Pharmacological interventions and

\section{Dovepress}

new therapies. Issues of patient safety and quality of care will also be considered. The manuscript management system is completely online and includes a very quick and fair peer-review system, which is all easy to use. Visit http://www.dovepress.com/testimonials.php to read real quotes from published authors. 